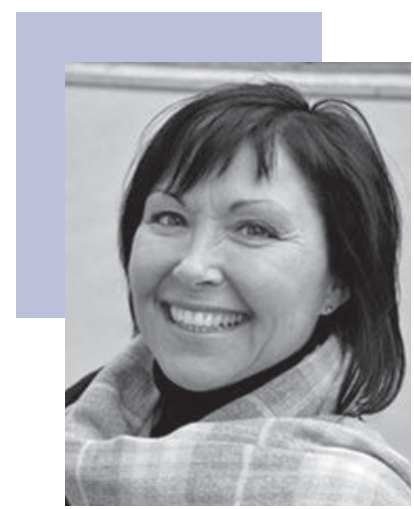

\title{
10 Jahre DMP - Disease Management Programme
}

Liebe Leserinnen und Leser,

Disease Management Programme nach SGB V $§ 137$ f sind strukturierte Behandlungsprogramme für chronisch Erkrankte. Die Behandlungs- und Betreuungsprozesse von Patienten werden über den gesamten Verlauf einer (chronischen) Krankheit koordiniert und auf der Grundlage wissenschaftlich gesicherter aktueller Erkenntnisse (medizinische Evidenz) optimiert. Wie das Kapitel SGB V ausweist, sind DMPs ausgewiesene Programme für Versicherte der Gesetzlichen Krankenversicherungen. Ziel ist die Verbesserung der Situation chronisch Erkrankter - insbesondere um Folgeschäden und Komplikationen der GKV-Versicherten zu vermeiden.

Es soll eine bedarfsgerechte und wirtschaftliche Versorgung sichergestellt werden - und eine Über-UnterFehlversorgung vermieden werden. Für die Zulassung der Programme ist das Bundesversicherungsamt zuständig. Zurzeit gibt es für jede der folgenden Krankheiten strukturierte Behandlungsprogramme:

> Diabetes mellitus Typ 1 seit 01.03.2004_ > Koronare Herzkrankheit seit 01.05.2003

> Diabetes mellitus Typ 2 seit 01.07.2002 >Asthma bronchiale seit 01.01.2005

$\begin{array}{lll}>\text { Brustkrebs } & \text { seit 01.07.2002 }>\text { COPD seit } 01.01 .2005\end{array}$

Zudem wurde am 01.07.2009 das Modul „chronische Herzinsuffizienz“ eingeführt. Aktuell sind rund 11000 Programme mit rund 6 Mio. Versicherten zugelassen. Soweit zum Anspruch dieser Programme. Die Realität nach 10 Jahren zeigt, dass die Anfrage und Nutzung dieser Programme mit 6 Mio. Versicherter der GKV auch in der Flächendeckung ein Erfolg ist. Bei genauerer fachwissenschaftlicher Sichtung besteht zu DMP-Programmen erheblicher Nachbesserungsbedarf. Diese Defizite traten zutage im Rahmen des Fach-Kongresses, den „Monitor Versorgungsforschung“ zusammen mit dem Bundesversicherungsamt (BVA) am 19.09.2011 in Berlin durchführte.

\section{Ergebnisse:}

$>$ Bei Einführung der DMPs vor 10 Jahren wurden sofort flächendeckende Programme eingeführt - völlig ohne Evidenz.

> Auch nach 10 Jahren (!) Realisation der Programme liegen keine ausreichenden und aussagekräftigen Evaluationen zu diesen Programmen vor.

> Studien zum Insulinbedarf weisen eine Hyperinsulinisierung der Versicherten seit Einführung der Programme aus.

$>$ Nutzen und Effizienz der Programme bleiben weiterhin fraglich.

$>$ Es gibt keine standardisierten Programme - die bestehenden folgen vielmehr dem Prinzip einer gewissen "Beliebigkeit“.

Aus Sicht der Fachverbandes DVGS gibt es noch 2 zusätzliche Problematiken:

1. Trotzdem alle genannten chronischen Erkrankungen vorwiegend durch Bewegungsmangelkrankheiten und durch falschen Lebensstil verursacht sind, fehlen standardisierte, biopsychosozial orientierte Bewegungsprogramme. Aus diesem Grund hat der DVGS für die Indikationen KHK, Diabetes mellitus Typ II, Brustkrebs und im Blick auf Co- und Multimorbiditäten auch das Thema Adipositas unter Federführung der GKV DAK solche konzeptioniert, bundesweit flächendeckend realisiert und auch evaluiert! Die Programme liegen derzeit dem Bundesversicherungsamt zur Akkreditierung vor.

2. Die DMPs als Versorgungsform werden künftig vielmehr auf multimorbides Klientel ausgerichtet werden müssen! Dabei ist eine geriatrisch-gerontologische Ausrichtung dringend notwendig. Die entsprechenden Versorgungsaspekte sollten sich zudem an den Klassifikationen der Gesundheitsdomänen der ICF (WHO) ressourcenorientiert - ausrichten. Zu dieser Zielsetzung kooperiert der DVGS sehr eng mit der Europäischen Vereinigung für Vitalität und aktives Altern (eVAA).

3. Werden die in Aspekt 1 und 2 genannten Optimierungen der DMPs nur mit interdisziplinären Ansätzen und multiprofessionell erreicht werden können. Eine Unterstützung des Haus- und Facharztes durch qualitätsgesicherte und professionalisierte Gesundheitsberufe wird unerlässlich sein.

In diesem Sinne entsteht - gerade durch die bestehenden Defizite innerhalb der Versorgungsform DMP - für unsere Berufsgruppe der Sport- und Bewegungstherapeuten ein sehr wichtiges Tätigkeitsfeld der Zukunft. Der DVGS hält für diese Tätigkeitsfelder sowohl die Programme für Leistungserbringer und Leistungsträger bereit - wie auch die qualifizierenden Curricula für die Gesundheitsberufe (siehe www.dvgs.de/neue Rubrik: Projekte).

Das vorliegende Heft informiert Sie, liebe Leserinnen und Leser, über diese aktuellen Programme, Projekte und Qualifikationsmöglichkeiten.

Viel Spaß bei der Lektüre wünscht Ihnen

Ihre Angelika Baldus 